Supplementary Information

\title{
Formation of PCDD/Fs in Oxidation of 2-Chlorophenol on Neat Silica Surface
}

\author{
Seyedehsara Mosallanejad ${ }^{1}$, Bogdan Z. Dlugogorski ${ }^{2}$, Eric M. Kennedy ${ }^{1}$, Michael \\ Stockenhuber ${ }^{1}$, Slawomir M. Lomnicki ${ }^{3}$, Niveen W. Assaf ${ }^{2}$ and Mohammednoor \\ Altarawneh $^{2}$
}

${ }^{1}$ Faculty of Engineering and Built Environment, The University of Newcastle, Callaghan, New South Wales 2308, Australia; ${ }^{2}$ School of Engineering and Information Technology, Murdoch University, Murdoch, WA 6150, Australia; ${ }^{3}$ Department of Environmental Sciences, Louisiana State University, Baton Rouge, Louisiana, USA

*Corresponding author: Phone: (+61) 893606364

Email: B.Dlugogorski@murdoch.edu.au

Page: 23

Figures: 6

Tables: 10 
Section 1. Impurities in the feed (2-CPh)

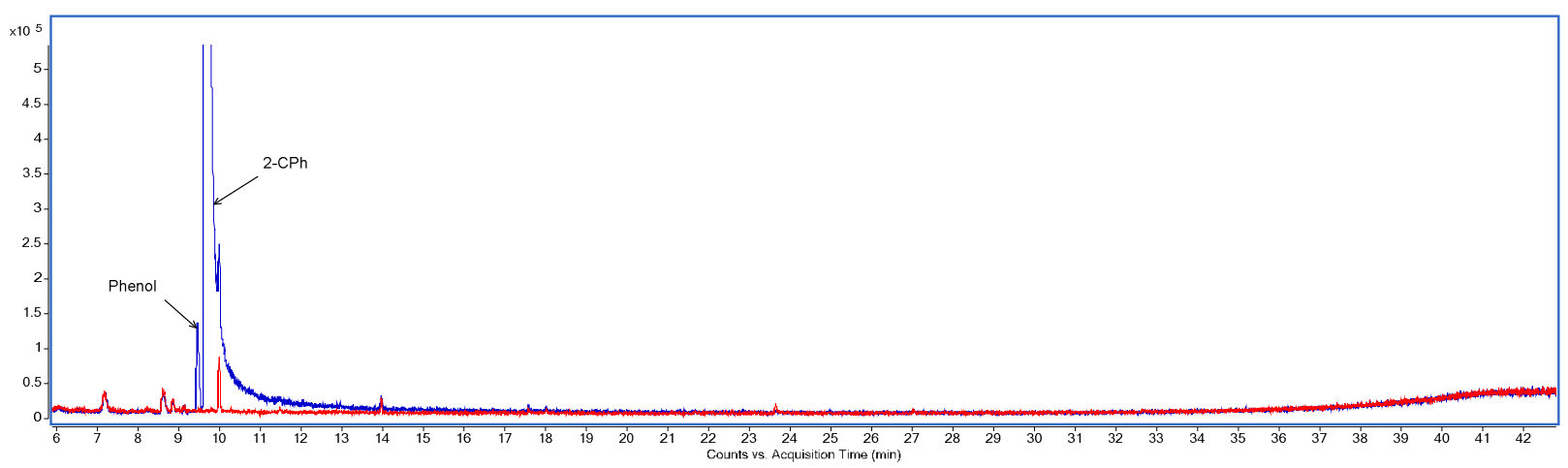

Figure S1. Comparison of 2-CPh solution in $n$-hexane with pure $n$-hexane; solution of 2$\mathrm{CPh}$ (blue line), $n$-hexane (red line) 
Section 2. ICP-OES

The elemental composition of the catalysts (silica gel) was measured using the inductively coupled plasma-optical emission spectrometry (Varian 715 ES spectrometer ICP-OES). Prior to the analysis, the samples were dissolved in a solution containing $4.5 \mathrm{~mL} \mathrm{HNO}_{3}(65 \%), 4.5$ $\mathrm{mL} \mathrm{HCl}(37 \%), 3 \mathrm{~mL} \mathrm{HBF}_{4}(50 \%)$ by Milestone Start D microwave unit for a minimum of $2 \mathrm{~h}$.

The percent fraction of silicon, the major element, is measured as $40.2 \% \pm 0.2 \%$.

Table S1. Elementary analysis of minor elements in silica gel

\begin{tabular}{lccc}
\hline Element & $\begin{array}{c}\text { Conc. } \\
{\left[\mu \mathbf{g ~ g}^{-1} \text { silica gel] }\right.}\end{array}$ & Element & $\begin{array}{c}\text { Conc. } \\
{\left[\mu \mathbf{g ~ g}^{-1} \text { silica gel] }\right.}\end{array}$ \\
\hline $\mathrm{Al}$ & n.d. & $\mathrm{Mg}$ & $204( \pm 3)$ \\
$\mathrm{Ba}$ & $8.6( \pm 0.3)$ & $\mathrm{Mn}$ & $8( \pm 2)$ \\
$\mathrm{Ca}$ & $890( \pm 30)$ & $\mathrm{Mo}$ & n.d. \\
$\mathrm{Cd}$ & n.d. & $\mathrm{Na}$ & $530( \pm 50)$ \\
$\mathrm{Co}$ & n.d. & $\mathrm{Ni}$ & n.d. \\
$\mathrm{Cr}$ & $7( \pm 3)$ & $\mathrm{Pb}$ & n.d. \\
$\mathrm{Cu}$ & n.d. & $\mathrm{Sr}$ & $3.9( \pm 0.4)$ \\
$\mathrm{Fe}$ & $71( \pm 6)$ & $\mathrm{Ti}$ & $96( \pm 1)$ \\
$\mathrm{K}$ & n.d. & $\mathrm{Zn}$ & n.d. \\
\hline
\end{tabular}

n.d.: Concentration below the detection limit $\left(2 \mu \mathrm{g} \mathrm{g}^{-1}\right)$ 
Section 3. Limit of detection and limit of quantitation of VOCs and mono-triCDD/Fs

To find the limits of detection (LOD) and the limits of quantitation (LOQ), we prepared a series of low concentration solutions of chlorophenols (CPhs), chlorobenzenes (CBzs) and mono-triCDD/Fs: (i) solutions of CPhs from purchased standard EPA 8270 phenol mix (Sigma Aldrich), (ii) solution of CBzs from purchased standard (AcuuStandard) and (iii) solutions of mono-triCDD/Fs from purchased standards (Wellington Laboratories and AccuStandard). LOD and LOQ for each compound correspond to a signal to noise ratio of 3 and 10 , respectively.

Table S2. Limits of detection and quantitation of CPhs

\begin{tabular}{lcc}
\hline Compounds & LOD $\left[\mathbf{n g ~} \mathbf{~} L^{\mathbf{- 1}}\right]$ & LOQ $\left[\mathbf{n g ~} \mathbf{~ m L}^{\mathbf{- 1}}\right]$ \\
\hline Phenol & 1.5 & 5.0 \\
2-CPh & 1.5 & 5.0 \\
2,4-DCPh & 2.5 & 8.0 \\
2,6-DCPh & 2.5 & 8.0 \\
2,4,6-TriCPh & 3 & 10.0 \\
\hline
\end{tabular}

Table S3. Limits of detection and quantitation of CBzs

\begin{tabular}{lcc}
\hline Compounds & LOD $\left[\mathbf{n g} \mathbf{~ m L}^{-\mathbf{1}}\right]$ & LOQ $\left[\mathbf{n g} \mathbf{~ m L}^{-1}\right]$ \\
\hline $\mathrm{Bz}$ & 1.1 & 3.0 \\
$\mathrm{CBz}$ & 1.0 & 3.2 \\
$1,4-\mathrm{DCBz}$ & 1.2 & 3.1 \\
$1,3-\mathrm{DCBz}$ & 1.0 & 3.0 \\
$1,2-\mathrm{DCBz}$ & 1.0 & 3.0 \\
$1,2,4-\mathrm{TriCBz}$ & 1.5 & 4.5 \\
$1,3,5-\mathrm{TriCBz}$ & 1.5 & 4.5 \\
$1,2,3-\mathrm{TriCBz}$ & 1.5 & 4.5 \\
$1,2,4,5-\mathrm{TeCBz}$ & 1.5 & 4.5 \\
$1,2,3,5-\mathrm{TeCBz}$ & 1.5 & 4.5 \\
$1,2,3,4-\mathrm{TeCBz}$ & 1.5 & 4.5 \\
$\mathrm{PeCBz}$ & 2.5 & 5.0 \\
$\mathrm{HCBz}$ & 2.5 & 5.0 \\
\hline
\end{tabular}


Table S4. Limits of detection and quantitation of mono-triCDD/Fs

\begin{tabular}{|c|c|c|}
\hline Compounds & LOD $\left[\mathrm{ng} \mathrm{mL} \mathrm{m}^{-1}\right]$ & LOQ $\left[\mathrm{ng} \mathrm{mL} \mathrm{m}^{-1}\right]$ \\
\hline$\overline{D D}$ & 0.3 & 1.0 \\
\hline $\mathrm{DF}$ & 0.15 & 0.5 \\
\hline 1-MCDD & 1.0 & 3.0 \\
\hline 4-MCDF & 1.0 & 3.0 \\
\hline 2,8-DCDF & 1.0 & 3.0 \\
\hline${ }^{13} \mathrm{C}_{12}-2,3-\mathrm{DCDF}$ & 1.0 & 3.0 \\
\hline 1,4-DCDD & 1.0 & 3.0 \\
\hline 1,6-DCDD & 1.0 & 3.0 \\
\hline 1,2-DCDD & 1.0 & 3.0 \\
\hline 1,3,6-TriCDF & 1.5 & 4.0 \\
\hline 1,2,3-TriCDD & 1.5 & 4.0 \\
\hline
\end{tabular}


Section 4. Recoveries of CPhs, CBzs and mono-triCDD/Fs from XAD-2 resin after a $2 \mathrm{~h}$ extraction with $n$-hexane by ultra-sonic bath. All analyses were repeated four times to calculate the standard error (SE).

Table S5. Recoveries of CPhs

\begin{tabular}{lc}
\hline CPhs & This study [\%] ( \pm SE) \\
\hline Phenol & $60.8( \pm 0.6)$ \\
2-CPh & $72.4( \pm 0.7)$ \\
$2,4-\mathrm{DCPh}$ & $53.4( \pm 0.5)$ \\
$2,6-\mathrm{DCPh}$ & $60.9( \pm 0.6)$ \\
$2,4,6-$ TriCPh & $53.3( \pm 0.4)$ \\
\hline
\end{tabular}

Table S6. Recoveries of CBzs

\begin{tabular}{lc}
\hline CBzs & This study $[\%]( \pm$ SE) \\
\hline $\mathrm{Bz}$ & $95.3( \pm 0.9)$ \\
$\mathrm{CBz}$ & $91.2( \pm 0.7)$ \\
$\mathrm{CBz}-\mathrm{d} 5$ & $90.5( \pm 0.8)$ \\
$1,3-\mathrm{DCBz}$ & $74.6( \pm 0.9)$ \\
$1,4-\mathrm{DCBz}$ & $86.5( \pm 0.8)$ \\
$1,2-\mathrm{DCBz}$ & $83.5( \pm 0.8)$ \\
$1,2,4-\mathrm{TriCBz}$ & $92.8( \pm 0.9)$ \\
$1,3,5-\mathrm{TriCBz}$ & $95.4( \pm 1.0)$ \\
$1,2,3-\mathrm{TriCBz}$ & $96.2( \pm 1.0)$ \\
$1,2,3,5-\mathrm{TeCBz}$ & $95.5( \pm 0.9)$ \\
$1,2,3,4-\mathrm{TeCBz}$ & $95.9( \pm 0.9)$ \\
PeCBz & $85.7( \pm 0.9)$ \\
$\mathrm{HexCBz}$ & $84.3( \pm 0.8)$ \\
\hline
\end{tabular}

These results indicate that more polar compounds are recovered less efficiently from the $\mathrm{XAD}-2$ resin in the ultrasonic bath. 
Table S7. Recoveries of DD/F and mono-triCDD/Fs

\begin{tabular}{lc}
\hline Congeners & This study $[\%]( \pm$ SE) \\
\hline $\mathrm{DD}$ & $71.9( \pm 0.7)$ \\
$\mathrm{DF}$ & $77.3( \pm 0.7)$ \\
$1-\mathrm{MCDD}$ & $70.7( \pm 0.7)$ \\
$4-\mathrm{MCDF}$ & $69.1( \pm 0.7)$ \\
$2,8-\mathrm{DCDF}$ & $63.3( \pm 0.6)$ \\
${ }^{13} \mathrm{C}_{12}-2,3-\mathrm{DCDF}$ & $68.9( \pm 0.7)$ \\
$1,4-\mathrm{DCDD}$ & $75.2( \pm 0.8)$ \\
$1,6-\mathrm{DCDD}$ & $68.5( \pm 0.7)$ \\
$1,2-\mathrm{DCDD}$ & $72.3( \pm 0.7)$ \\
1,3,6-TriCDF & $77.1( \pm 0.8)$ \\
1,2,3-TriCDD & $73.2( \pm 0.8)$ \\
\hline
\end{tabular}


Section 5. Yields of $\mathrm{CPhs}$ and mono-diCDD/Fs, at reaction temperatures of 350 and $400{ }^{\circ} \mathrm{C}$

The reaction at $350{ }^{\circ} \mathrm{C}$ was repeated three times to calculate the standard error (SE); standard error and standard deviation are related by the following formula $\mathrm{SE}=\mathrm{SD} /$ (number of experiments) $)^{1 / 2}$

We calculated the conversion of 2-CPh based on the following formula

2-CPh conversion $=\frac{C_{2-C P h}^{i n}-C_{2-C P h}^{o u t}}{C_{2-C P h}^{i n}}$, where $C$ denotes concentration, in moles per unit volume

The 2-CPh conversion allows the calculation of yield with an aid of a simple expression

Yield $=2-\mathrm{CPh}$ conversion $\times$ selectivity, where selectivity is defined as

Selectivity $=\frac{C_{P \text { roduct }}^{\text {out }} \times A}{C_{2-C P h}^{\text {in }}-C_{2-C P h}^{\text {out }}} \quad(A=2$ for PCDD/Fs and $A=1$ for $\mathrm{CPhs})$

Table S8. Yields of CPhs identified in the current study

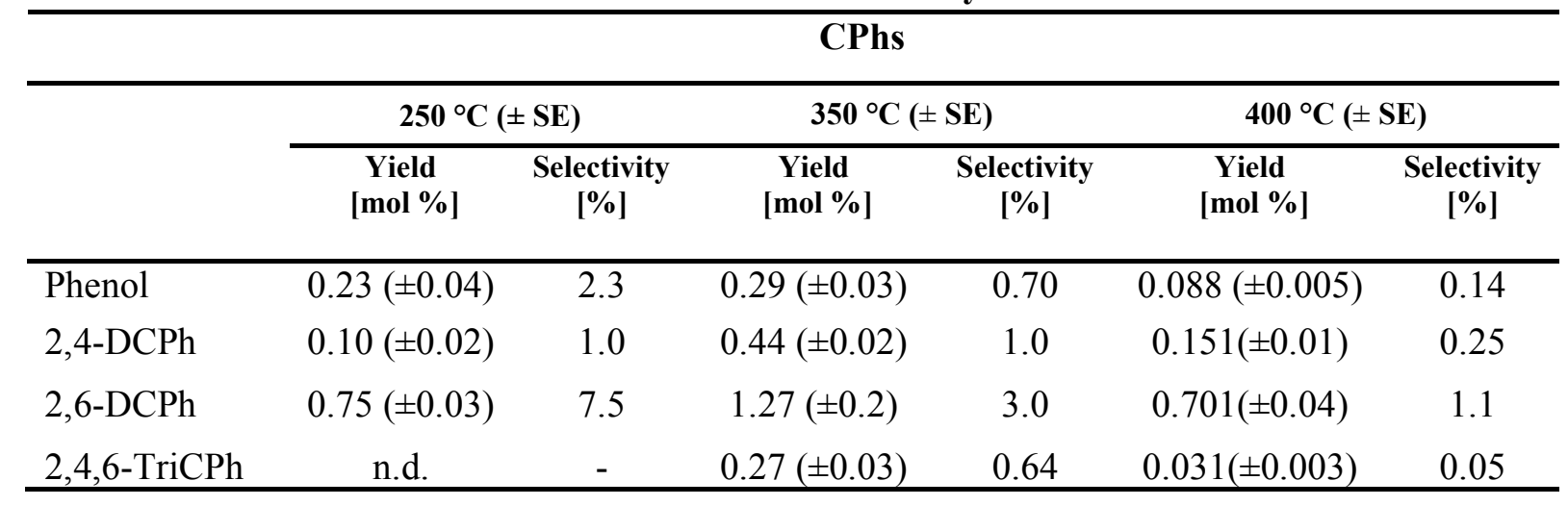

n.d.: not detected

We detected no chlorobenzenes in experiments involving the neat silica catalyst 
Table S9. Yields of mono-diCDD/Fs identified in the current study

\begin{tabular}{|c|c|c|c|c|c|}
\hline \multicolumn{6}{|c|}{ Congeners } \\
\hline & \multirow{2}{*}{$\begin{array}{c}250^{\circ} \mathrm{C} \\
\begin{array}{c}\text { Yield } \\
{[\text { mol \%] }}\end{array}\end{array}$} & \multicolumn{2}{|c|}{$3500^{\circ} \mathrm{C}( \pm \mathrm{SE})$} & \multicolumn{2}{|c|}{$400^{\circ} \mathrm{C}( \pm \mathrm{SE})$} \\
\hline & & $\begin{array}{c}\text { Yield } \\
\text { [mol \%] }\end{array}$ & $\begin{array}{c}\text { Selectivity } \\
{[\%]}\end{array}$ & $\begin{array}{c}\text { Yield } \\
\text { [mol \%] }\end{array}$ & $\begin{array}{c}\text { Selectivity } \\
{[\%]}\end{array}$ \\
\hline DD & n.d. & $0.021( \pm 0.004)$ & 0.02 & $0.032( \pm 0.005)$ & 0.05 \\
\hline 1-MCDD & n.d. & $0.066( \pm 0.003)$ & 0.07 & $0.041( \pm 0.003)$ & 0.07 \\
\hline 2-MCDD & n.d. & $0.0052( \pm 0.0004)$ & 0.005 & $0.003( \pm 0.0005)$ & 0.005 \\
\hline 4-MCDF & n.d. & $0.0052( \pm 0.0005)$ & 0.005 & $0.007( \pm 0.0003)$ & 0.01 \\
\hline 1,3-DCDD & n.d. & $0.0020( \pm 0.0002)$ & 0.002 & $0.0005( \pm 0.0001)$ & 0.0008 \\
\hline 1,6-DCDD & n.d. & $0.0068( \pm 0.0003)$ & 0.007 & $0.003( \pm 0.0004)$ & 0.005 \\
\hline 1,9-DCDD & n.d. & $0.0032( \pm 0.0002)$ & 0.003 & $0.001( \pm 0.0002)$ & 0.002 \\
\hline 4,6-DCDF & n.d. & $0.021( \pm 0.004)$ & 0.02 & $0.012( \pm 0.003)$ & 0.02 \\
\hline
\end{tabular}

n.d.: not detected 
Section 6- Comparison between the yields of dioxin congeners from experiments involving iron and copper oxide-silica supported catalysts

Table S10. Comparison between the dioxins yields of this study with the two previous studies.

\begin{tabular}{|c|c|c|c|c|c|c|}
\hline Study & Reactant & Catalyst & Environment & $\begin{array}{c}\text { Identified/quantitated } \\
\text { products }\end{array}$ & $\begin{array}{l}\text { Yield at } \\
3500^{\circ} \mathrm{C}\end{array}$ & $\begin{array}{c}\text { Yield in previous } \\
\text { studies/yield in the } \\
\text { present study (from } \\
\text { Table S9) }\end{array}$ \\
\hline \multirow{4}{*}{ Nganai et al. ${ }^{1}$} & \multirow{4}{*}{ 2-CPh } & \multirow{4}{*}{$\begin{array}{l}\text { Iron } \\
\text { oxide on } \\
\text { silica }\end{array}$} & \multirow{4}{*}{ Oxidation } & DD & 0.048 & 2.3 \\
\hline & & & & 1-MCDD & 0.45 & 6.8 \\
\hline & & & & DF & 0.1 & -- \\
\hline & & & & $4,6-\mathrm{DCDF}$ & 0.36 & 17.1 \\
\hline \multirow{3}{*}{$\begin{array}{c}\text { Lomnicki et } \\
\text { al. }^{2}\end{array}$} & \multirow{3}{*}{ 2-CPh } & \multirow{3}{*}{$\begin{array}{l}\text { Copper } \\
\text { oxide on } \\
\text { silica }\end{array}$} & \multirow{3}{*}{ Oxidation } & DD & 0.07 & 3.3 \\
\hline & & & & 1-MCDD & 0.28 & 4.2 \\
\hline & & & & $4,6-\mathrm{DCDF}$ & 0.1 & 5.0 \\
\hline
\end{tabular}


Section 7. Extracted ion chromatogram (EIC) for the experiments conducted at 250, 350, $400{ }^{\circ} \mathrm{C}$ and for a genuine standard

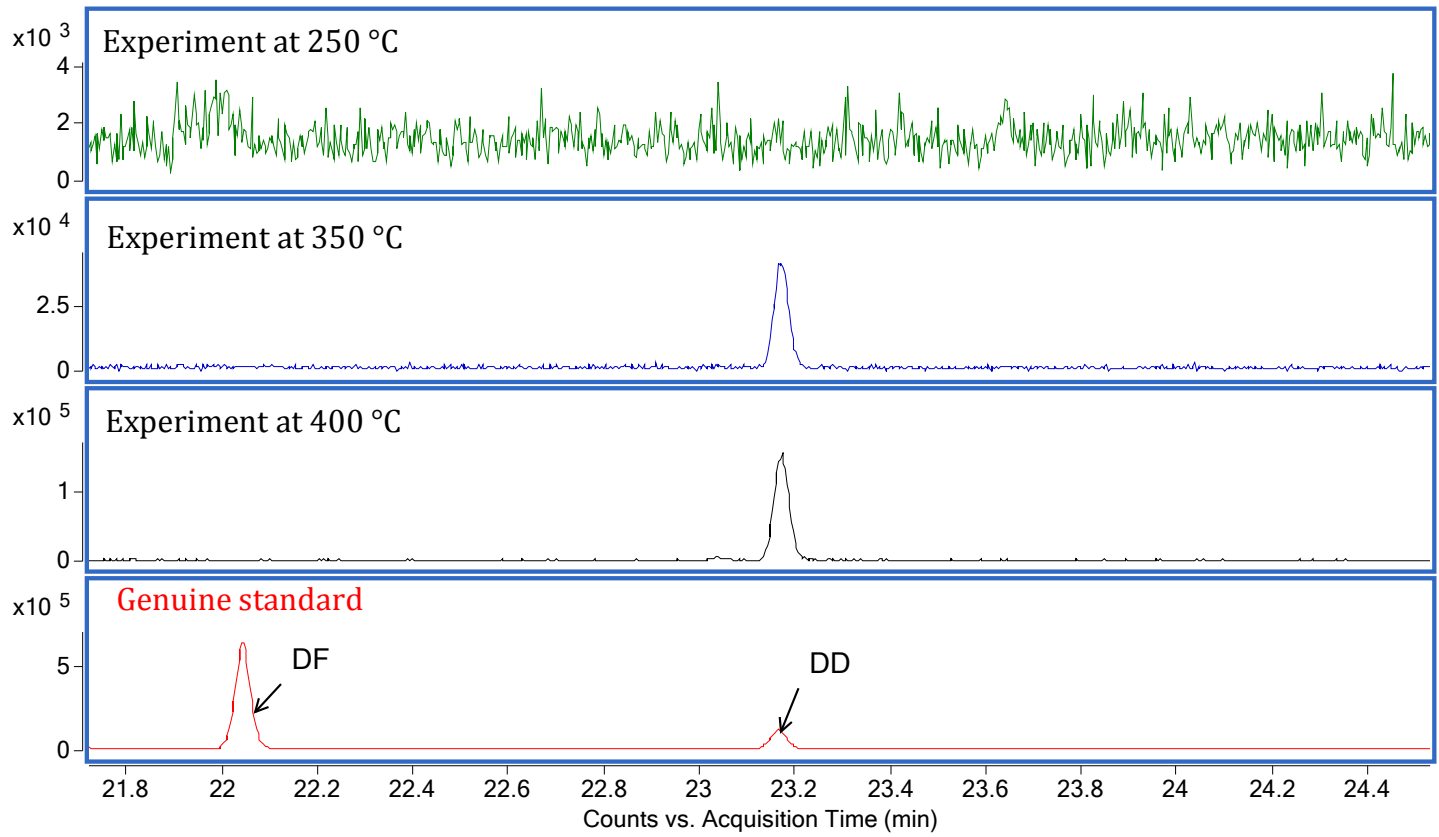

Figure S2. Extracted ion chromatogram (EIC) of the sample from experiment conducted at $250{ }^{\circ} \mathrm{C}, 350{ }^{\circ} \mathrm{C}$ and $400{ }^{\circ} \mathrm{C}$ compared with a trace of the genuine standard; DF $(m / z=168.0573,139.0555)$ and DD $(m / z=184.0538,128.0638)$. 


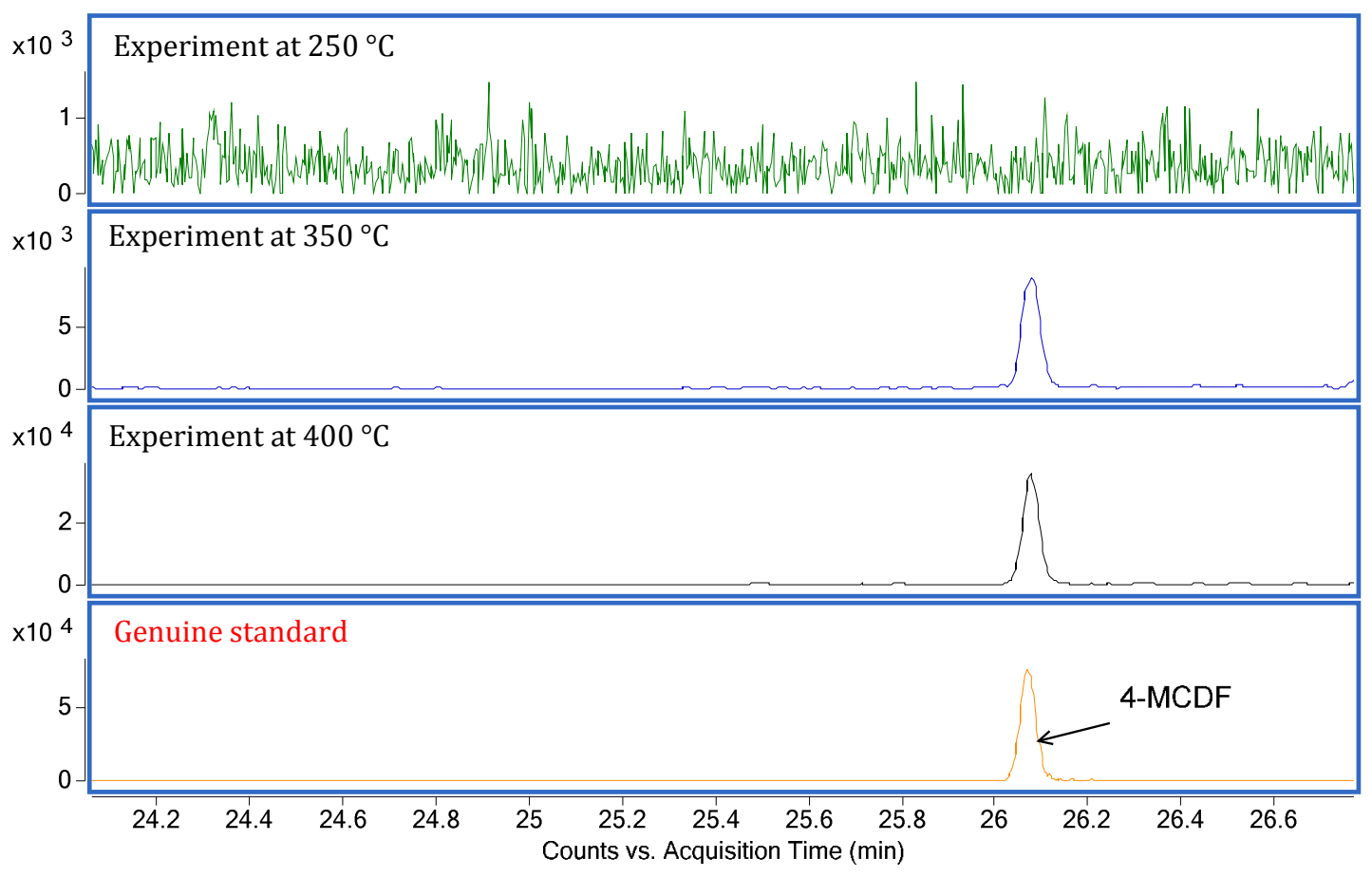

Figure S3. Extracted ion chromatogram (EIC) of the sample from the experiment conducted at $250{ }^{\circ} \mathrm{c}, 350{ }^{\circ} \mathrm{C}$ and $400{ }^{\circ} \mathrm{C}$ compared with a trace of the genuine standard; MCDF $(\mathrm{m} / \mathrm{z}=202.0190,139.0555)$.

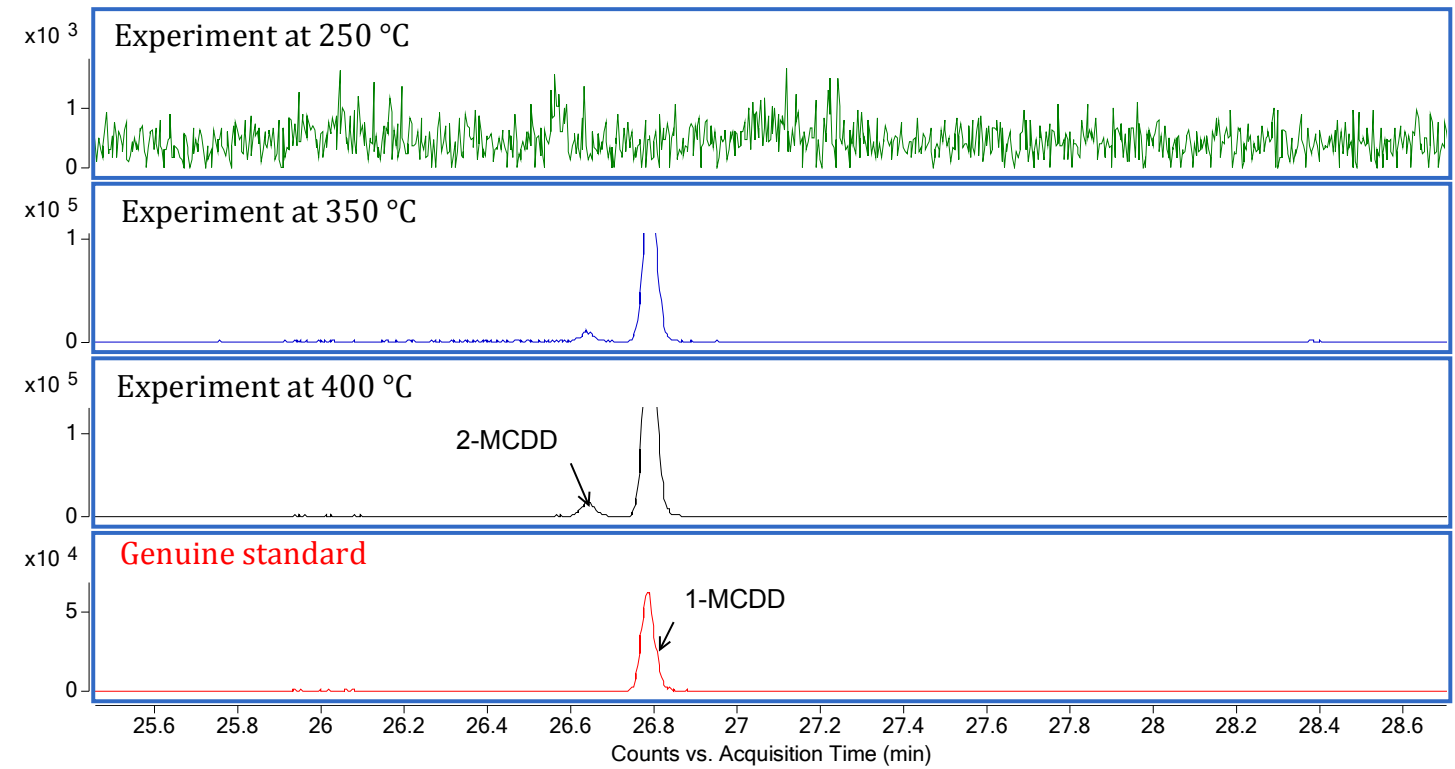

Figure S4. Extracted ion chromatogram (EIC) of the sample from the experiment conducted at $250{ }^{\circ} \mathrm{C}, 350{ }^{\circ} \mathrm{C}, 400{ }^{\circ} \mathrm{C}$ and a segment of the genuine standard for MCDD; MCDD $(\mathrm{m} / \mathrm{z}=218.0142,155.0503)$. 


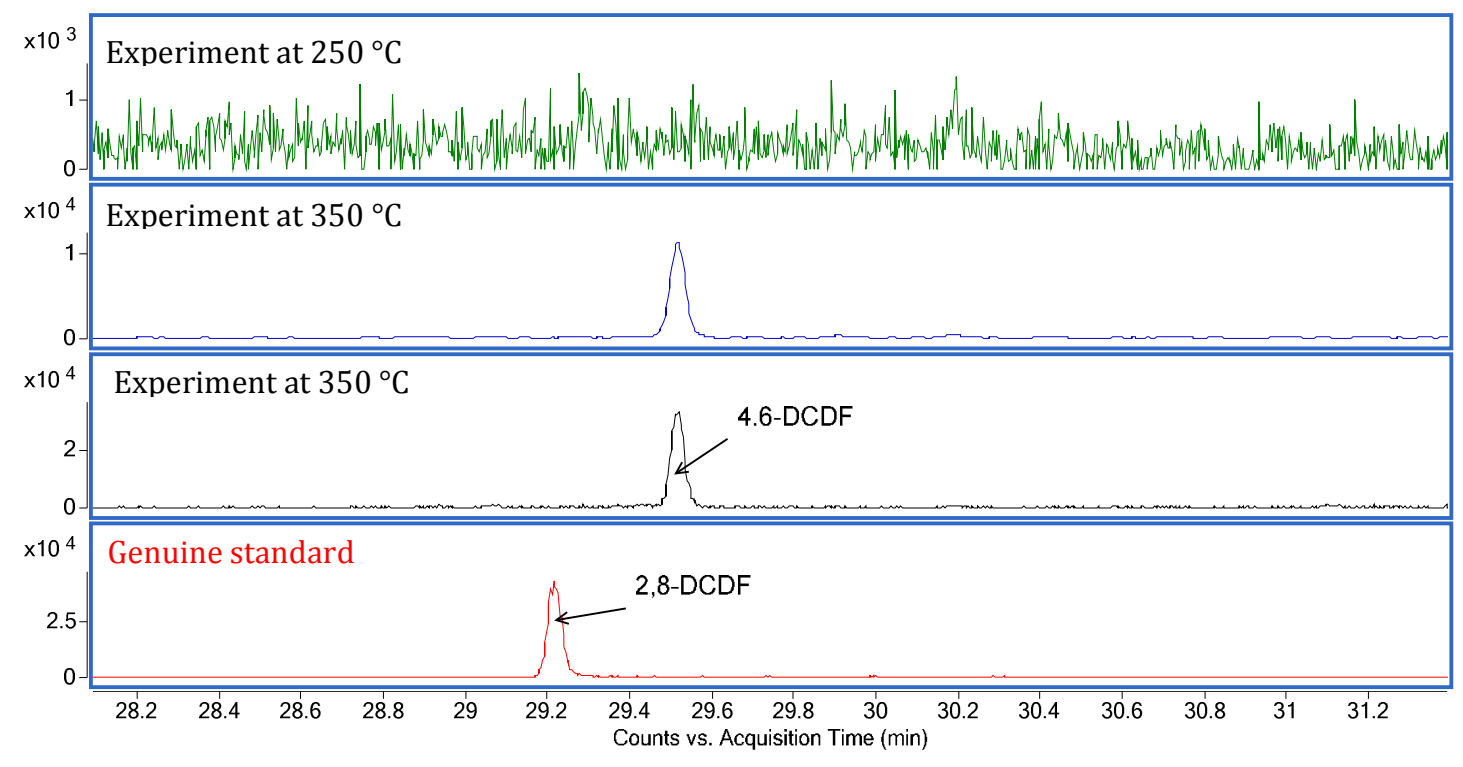

Figure S5. Extracted ion chromatogram (EIC) of the sample from the experiment conducted at $250{ }^{\circ} \mathrm{C}, 350{ }^{\circ} \mathrm{C}$ and $400{ }^{\circ} \mathrm{C}$ compared with a segment of the genuine standard for DCDF; $\operatorname{DCDF}(\mathrm{m} / z=235.9803,173.0172)$.

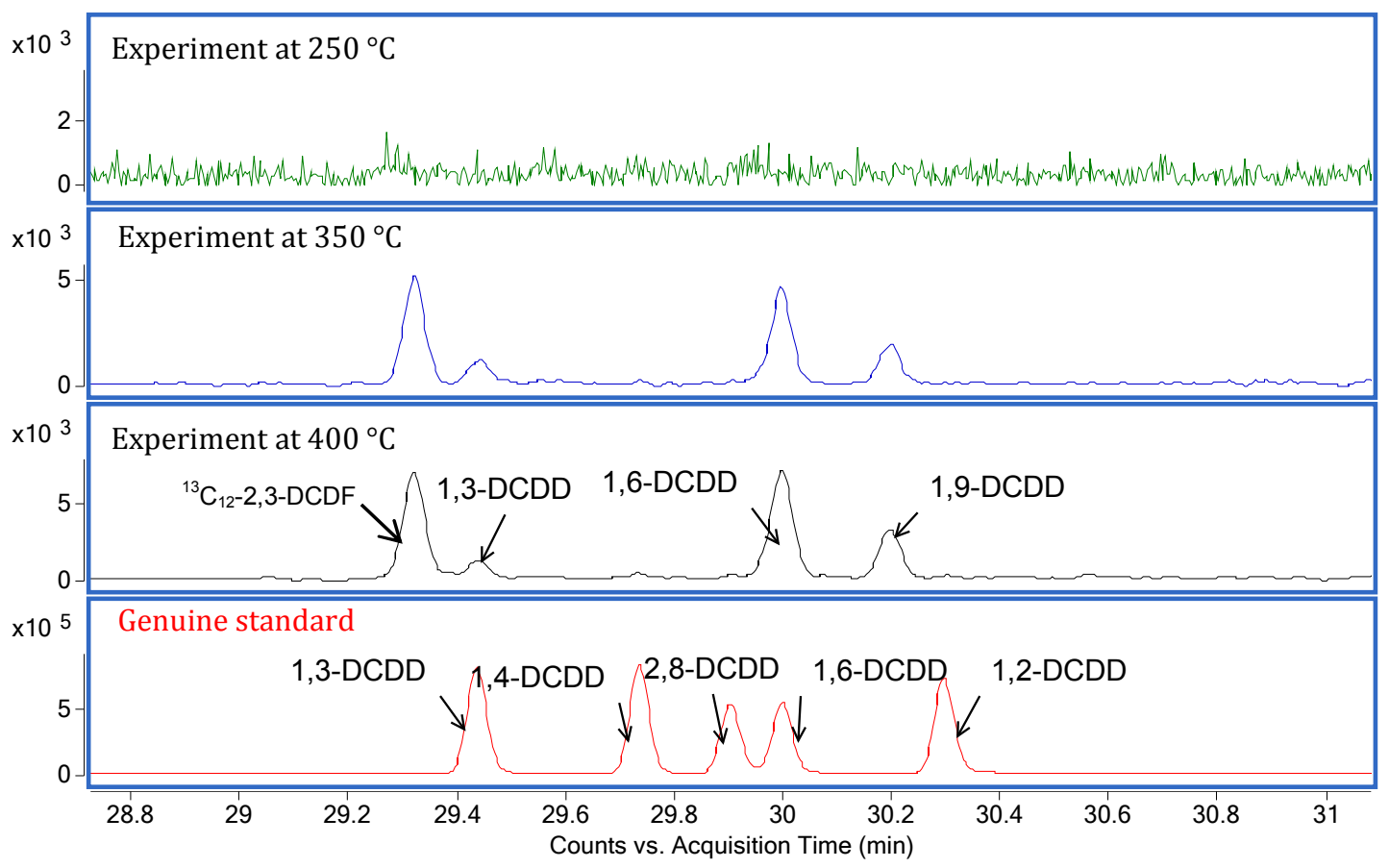

Figure S6. Extracted ion chromatogram (EIC) of the sample from the experiment conducted at $250{ }^{\circ} \mathrm{C}, 350{ }^{\circ} \mathrm{C}$ and $400{ }^{\circ} \mathrm{C}$ compared with a segment of the genuine standard for DCDD; $\operatorname{DCDD}(m / z=251.8977,188.9441)$. 
Section 8. Cartesian coordinates for all structures involved in this study

$\begin{array}{llll}\text { M1 } & & & \\ \mathrm{C} & -0.47260 & 2.217057 & -0.12319 \\ \mathrm{C} & 0.378290 & 3.325020 & -0.11316 \\ \mathrm{C} & 1.764273 & 3.145796 & -0.07582 \\ \mathrm{C} & 2.285324 & 1.851068 & -0.04580 \\ \mathrm{C} & 1.423141 & 0.749319 & -0.05577 \\ \mathrm{C} & 0.045208 & 0.917618 & -0.09573 \\ \mathrm{O} & 1.993072 & -0.54067 & -0.02730 \\ \mathrm{H} & -1.55395 & 2.351882 & -0.15654 \\ \mathrm{H} & -0.03389 & 4.334003 & -0.13307 \\ \mathrm{H} & 2.446978 & 3.995200 & -0.06744 \\ \mathrm{H} & -0.63213 & 0.067947 & -0.10879 \\ \mathrm{H} & 2.983691 & -0.50049 & -0.00249 \\ \mathrm{Cl} & 4.007032 & 1.566919 & 0.005551 \\ \mathrm{Si} & -3.16128 & -3.01704 & -0.00220 \\ \mathrm{Si} & -0.79004 & -2.71697 & 0.023914 \\ \mathrm{Si} & 1.574600 & -2.41743 & 0.049963 \\ \mathrm{O} & -2.13391 & -1.68431 & -0.03142 \\ \mathrm{O} & 0.331713 & -2.49237 & 1.225414 \\ \mathrm{O} & -1.83470 & -4.04346 & 0.051948 \\ \mathrm{O} & 0.365391 & -2.56946 & -1.15501 \\ \mathrm{O} & 3.010248 & -2.94310 & 0.084102 \\ \mathrm{O} & -4.67345 & -3.19511 & -0.01684\end{array}$

TS1

C

C

C

C

C

C

$\mathrm{O}$

$\mathrm{H}$

$\mathrm{H}$

$\mathrm{H}$

$\mathrm{H}$

$\mathrm{H}$

$\mathrm{Cl}$

$\mathrm{Si}$

$\mathrm{Si}$

$\mathrm{Si}$

$\mathrm{O}$

$\mathrm{O}$

O

$\begin{array}{lll}-0.70924 & 2.309994 & -0.29107 \\ 0.148979 & 3.378607 & -0.00398 \\ 1.507107 & 3.133674 & 0.208833 \\ 1.999757 & 1.825936 & 0.160333 \\ 1.141875 & 0.771259 & -0.16945 \\ -0.209330 & 1.009478 & -0.42975 \\ 1.642244 & -0.53233 & -0.29003 \\ -1.777390 & 2.496976 & -0.44626 \\ -0.230610 & 4.405032 & 0.061083 \\ 2.198104 & 3.954163 & 0.413537 \\ -0.890630 & 0.224343 & -0.78041 \\ 2.655974 & -1.09034 & -0.29920 \\ 3.665876 & 1.495433 & 0.526297 \\ -3.599650 & -2.83621 & 0.265411 \\ -1.223520 & -2.55765 & 0.164322 \\ 1.1215300 & -2.21145 & 0.012008 \\ -2.560440 & -1.52789 & 0.030667 \\ -0.025020 & -2.20919 & 1.266354 \\ -2.268160 & -3.86353 & 0.396148\end{array}$




$\begin{array}{llll}\mathrm{O} & -0.125580 & -2.57204 & -1.08221 \\ \mathrm{O} & 2.6419880 & -2.59578 & -0.03771 \\ \mathrm{O} & -5.103880 & -3.00849 & 0.325073\end{array}$

M2

C

C

C

C

C

C

$\mathrm{O}$

$\mathrm{H}$

$\mathrm{H}$

$\mathrm{H}$

$\mathrm{H}$

$\mathrm{H}$

$\mathrm{Cl}$

$\mathrm{Si}$

$\mathrm{Si}$

$\mathrm{Si}$

$\mathrm{O}$

$\mathrm{O}$

$\mathrm{O}$

O

$\mathrm{O}$

$\mathrm{O}$

$\begin{array}{lll}0.305273 & 1.889739 & -0.85162 \\ 1.014895 & 2.683836 & 0.055132 \\ 2.169727 & 2.188875 & 0.667981 \\ 2.613754 & 0.896619 & 0.374711 \\ 1.905647 & 0.101960 & -0.54079 \\ 0.753342 & 0.600076 & -1.15335 \\ 2.377146 & -1.16815 & -0.83979 \\ -0.59841 & 2.271406 & -1.32830 \\ 0.67381 & 3.692789 & 0.289856 \\ 2.734144 & 2.795536 & 1.376248 \\ 0.220754 & -0.03150 & -1.86686 \\ 3.615718 & -3.53065 & -0.38842 \\ 4.032573 & 0.259435 & 1.156206 \\ -2.97697 & -2.87008 & 0.810404 \\ -0.66969 & -2.71890 & 0.229931 \\ 1.613895 & -2.54593 & -0.38095 \\ -1.96885 & -1.63785 & 0.272159 \\ 0.727250 & -2.37435 & 1.061362 \\ -1.68885 & -3.94911 & 0.774198 \\ 0.168884 & -2.90282 & -1.19623 \\ 2.677135 & -3.77007 & -0.46433 \\ -4.45298 & -2.96969 & 1.172766\end{array}$

M3

C

C

C

C

C

C

O

$\mathrm{H}$

$\mathrm{H}$

$\mathrm{H}$

H

$\mathrm{Si}$

$\mathrm{Si}$

$\mathrm{Si}$

O

O

$\begin{array}{lll}-4.37469 & 6.48073 & 0.584173 \\ -5.68700 & 6.943892 & 0.422130 \\ -6.74985 & 6.044221 & 0.533384 \\ -6.49549 & 4.698539 & 0.803512 \\ -5.18196 & 4.214964 & 0.966129 \\ -4.12608 & 5.136173 & 0.851367 \\ -4.86245 & 2.927068 & 1.236567 \\ -3.53565 & 7.173732 & 0.500190 \\ -5.88669 & 7.99538 & 0.213313 \\ -7.78161 & 6.377688 & 0.417108 \\ -3.11087 & 4.754937 & 0.971577 \\ -5.45092 & -0.43129 & -0.13003 \\ -3.07703 & -0.47129 & 0.003766 \\ -0.71573 & -0.59723 & 0.131557 \\ -4.32583 & -1.09276 & 0.935311 \\ -1.89168 & 0.469701 & 0.710171\end{array}$




$\begin{array}{llll}\mathrm{O} & -4.18275 & 0.190262 & -1.06600 \\ \mathrm{O} & -1.92294 & -1.53195 & -0.59102 \\ \mathrm{O} & 0.074896 & -1.50833 & 1.242717 \\ \mathrm{H} & 0.815551 & -1.09011 & 1.709466 \\ \mathrm{O} & -6.46175 & -1.43399 & -0.93894 \\ \mathrm{H} & -6.05408 & -2.03680 & -1.58218 \\ \mathrm{O} & -6.40054 & 0.664200 & 0.637569 \\ \mathrm{H} & -7.27549 & 0.847664 & 0.257041 \\ \mathrm{O} & 0.303844 & 0.209405 & -0.86806 \\ \mathrm{H} & 1.075027 & -0.28164 & -1.19323 \\ \mathrm{H} & -5.64446 & 2.335104 & 1.160294 \\ \mathrm{Cl} & -7.85315 & 3.591205 & 0.966358\end{array}$

\section{TS2}

C

C

C

C

C

C

$\mathrm{O}$

$\mathrm{H}$

$\mathrm{H}$

$\mathrm{H}$

$\mathrm{H}$

$\mathrm{Si}$

$\mathrm{Si}$

$\mathrm{Si}$

O

$\mathrm{O}$

O

O

$\mathrm{O}$

$\mathrm{H}$

$\mathrm{O}$

$\mathrm{H}$

$\mathrm{O}$

$\mathrm{H}$

O

$\mathrm{H}$

$\mathrm{H}$

$\mathrm{Cl}$

M4
$-4.75268$

$-5.6041$

$-6.6386$

$-6.78151$

$-5.91672$

$-4.91041$

$-6.12358$

$-3.94789$

$-5.47854$

$-7.34226$

$-4.32725$

$-5.86197$

$-3.50432$

$-1.17571$

$-4.61717$

$-2.17322$

$-4.67733$

$-2.50073$

$-0.16714$

0.693578

$-6.50231$

$-5.82862$

$-7.50273$

$-8.18688$

$-0.38489$

0.455999

$-7.29269$

$-8.07017$
6.106058

6.812422

6.135399

4.759158

4.047743

4.730283

2.717358

6.607506

7.879965

6.663816

4.142741

0.953745

0.917843

0.698524

0.403080

1.839277

1.540246

$-0.25311$

$-0.17940$

0.256374

$-0.36298$

$-0.87968$

1.03112

0.775815

1.422156

0.965492

2.153358

3.865074
1.345449

0.478372

$-0.17745$

$-0.00816$

0.830718

1.52143

1.091718

1.889465

0.299605

$-0.81603$

2.247807

0.302492

0.178674

$-0.13165$

1.309762

0.614182

$-0.80223$

$-0.50275$

0.809972

0.977019

$-0.53136$

$-1.04185$

1.11255

0.447325

$-1.37317$

$-1.58235$

1.191506

$-0.79705$ 


$\begin{array}{llll}\mathrm{C} & -6.00856 & 7.042723 & 0.862357 \\ \mathrm{C} & -7.08910 & 6.609280 & 0.090669 \\ \mathrm{C} & -7.39760 & 5.248574 & 0.027389 \\ \mathrm{C} & -6.62614 & 4.316980 & 0.742006 \\ \mathrm{C} & -5.55212 & 4.756130 & 1.521002 \\ \mathrm{O} & -6.98725 & 2.981932 & 0.691687 \\ \mathrm{H} & -4.39664 & 6.449409 & 2.182065 \\ \mathrm{H} & -5.77028 & 8.106623 & 0.903204 \\ \mathrm{H} & -7.69781 & 7.315874 & -0.47357 \\ \mathrm{H} & -4.97252 & 4.02342 & 2.084935 \\ \mathrm{Si} & -5.97790 & 1.751492 & 0.303984 \\ \mathrm{Si} & -3.61770 & 1.864402 & 0.013619 \\ \mathrm{Si} & -1.26760 & 1.964509 & -0.26852 \\ \mathrm{O} & -4.63173 & 1.547098 & 1.311938 \\ \mathrm{O} & -2.47589 & 3.076332 & 0.144002 \\ \mathrm{O} & -4.94137 & 2.053861 & -0.99465 \\ \mathrm{O} & -2.43814 & 0.750112 & -0.39203 \\ \mathrm{O} & -0.20084 & 1.567173 & 0.909326 \\ \mathrm{H} & 0.581816 & 2.132098 & 1.007780 \\ \mathrm{O} & -6.99225 & 0.478040 & 0.188282 \\ \mathrm{H} & -6.59801 & -0.40097 & 0.069061 \\ \mathrm{O} & -9.89669 & 1.585232 & 0.340657 \\ \mathrm{H} & -9.09583 & 1.033788 & 0.308764 \\ \mathrm{O} & -0.54420 & 2.445978 & -1.65720 \\ \mathrm{H} & 0.199659 & 1.912879 & -1.97986 \\ \mathrm{H} & -9.53276 & 2.486867 & 0.315844 \\ \mathrm{Cl} & -8.73553 & 4.70431 & -0.94308 \\ & & & \end{array}$

M5

C

C

C

C

C

C

C

C

C

C

C

C

O

$\mathrm{O}$

$\mathrm{H}$

$\mathrm{H}$

$-5.49314$

6.148790

1.161764

$-13.9835$

9.293119

$-0.10973$

$-6.50195$

6.967926

0.646044

$-15.0157$

8.345660

$-0.12456$

$-7.67639$

6.400468

0.144059

$-14.7084$

6.993833

$-0.30184$

$-7.83109$

5.011848

0.147639

$-13.3776$

6.601802

$-0.45883$

$-6.82059$

4.183058

0.664899

$-12.3298$

7.543125

$-0.44116$

$-5.65571$

4.760803

1.180259

$-12.6546$

8.896749

$-0.26637$

$-7.03879$

2.822166

0.675482

$-11.0175$

7.208286

$-0.58656$

$-4.57595$

6.586064

1.558591

$-14.2139$

10.35101

0.027020 


$\begin{array}{llll}\mathrm{H} & -6.38095 & 8.051905 & 0.634103 \\ \mathrm{H} & -16.0553 & 8.65254 & 0.000323 \\ \mathrm{H} & -8.48067 & 7.02543 & -0.24593 \\ \mathrm{H} & -15.4923 & 6.236734 & -0.31888 \\ \mathrm{H} & -4.88943 & 4.116949 & 1.614252 \\ \mathrm{H} & -11.8398 & 9.621573 & -0.25775 \\ \mathrm{H} & -10.9324 & 6.23293 & -0.64778 \\ \mathrm{Si} & -5.9694 & 1.620836 & 0.376874 \\ \mathrm{Si} & -3.61467 & 1.815206 & 0.062064 \\ \mathrm{Si} & -1.26726 & 1.990784 & -0.20898 \\ \mathrm{O} & -4.6068 & 1.570206 & 1.388325 \\ \mathrm{O} & -2.52279 & 3.082103 & 0.097077 \\ \mathrm{O} & -4.94480 & 1.867200 & -0.95068 \\ \mathrm{O} & -2.38554 & 0.724280 & -0.25353 \\ \mathrm{O} & -0.20464 & 1.723715 & 1.01063 \\ \mathrm{H} & 0.516448 & 2.363921 & 1.118987 \\ \mathrm{O} & -6.89948 & 0.285224 & 0.360744 \\ \mathrm{H} & -6.43707 & -0.55996 & 0.244727 \\ \mathrm{O} & -0.53449 & 2.390300 & -1.61875 \\ \mathrm{H} & 0.261165 & 1.890376 & -1.86053 \\ \mathrm{Cl} & -9.28331 & 4.289381 & -0.49465 \\ \mathrm{Cl} & -12.9794 & 4.909572 & -0.68573\end{array}$

TS3

C

C

C

C

C

C

C

C

C

C

C

C

$\mathrm{O}$

$\mathrm{O}$

$\mathrm{H}$

H

$\mathrm{H}$

$\mathrm{H}$

H

$\mathrm{H}$

$\mathrm{H}$

$\begin{array}{lll}-5.24566 & 6.337741 & 1.705381 \\ -11.2668 & 6.019819 & 1.445474 \\ -5.84556 & 7.299262 & 0.88292 \\ -12.2641 & 6.312161 & 0.510696 \\ -6.91091 & 6.932567 & 0.049565 \\ -12.037 & 5.991889 & -0.82984 \\ -7.36637 & 5.610443 & 0.011288 \\ -10.7757 & 5.564250 & -1.25159 \\ -6.71397 & 4.633429 & 0.800026 \\ -9.70621 & 5.488862 & -0.34022 \\ -5.69150 & 5.017372 & 1.680949 \\ -10.0051 & 5.584391 & 1.022415 \\ -7.13608 & 3.323011 & 0.755537 \\ -8.42650 & 5.275840 & -0.82843 \\ -4.41307 & 6.622502 & 2.363384 \\ -11.4615 & 6.098618 & 2.511585 \\ -5.50198 & 8.335756 & 0.901346 \\ -13.2296 & 6.716714 & 0.828448 \\ -7.41641 & 7.669276 & -0.57328 \\ -12.8658 & 6.012236 & -1.54901 \\ -5.26359 & 4.256023 & 2.34583\end{array}$




$\begin{array}{llll}\mathrm{H} & -9.23097 & 5.300068 & 1.735979 \\ \mathrm{H} & -5.05027 & 13.56606 & -4.70108 \\ \mathrm{Si} & -6.16731 & 2.051031 & 0.379043 \\ \mathrm{Si} & -3.77546 & 1.98256 & 0.068886 \\ \mathrm{Si} & -1.39829 & 1.909042 & -0.13609 \\ \mathrm{O} & -4.81825 & 1.74784 & 1.37204 \\ \mathrm{O} & -2.54443 & 3.105938 & 0.18710 \\ \mathrm{O} & -5.0995 & 2.272897 & -0.92069 \\ \mathrm{O} & -2.65834 & 0.788515 & -0.31377 \\ \mathrm{O} & -0.42611 & 1.463694 & 1.114236 \\ \mathrm{H} & 0.417408 & 1.960592 & 1.19054 \\ \mathrm{O} & -7.25713 & 0.845006 & 0.290226 \\ \mathrm{H} & -6.9049 & -0.05915 & 0.192609 \\ \mathrm{O} & -0.51216 & 2.336941 & -1.45531 \\ \mathrm{H} & 0.218922 & 1.776423 & -1.76655 \\ \mathrm{Cl} & -4.49299 & 12.58345 & -4.05944 \\ \mathrm{Cl} & -10.504 & 5.066382 & -2.90343\end{array}$

M6

C

C

C

C

C

C

C

C

C

C

C

C

O

$\mathrm{O}$

$\mathrm{H}$

$\mathrm{H}$

$\mathrm{H}$

$\mathrm{H}$

$\mathrm{H}$

$\mathrm{H}$

$\mathrm{H}$

$\mathrm{H}$

$\mathrm{H}$

$\mathrm{Si}$

$\mathrm{Si}$

$\mathrm{Si}$

$\begin{array}{lll}-5.09002 & 6.434651 & 1.291277 \\ -11.3225 & 6.697698 & 0.838415 \\ -5.83215 & 7.293185 & 0.473374 \\ -12.2946 & 6.485247 & -0.14372 \\ -6.97013 & 6.818488 & -0.18494 \\ -11.9666 & 5.774342 & -1.30130 \\ -7.36604 & 5.487848 & -0.02444 \\ -10.6718 & 5.282231 & -1.48197 \\ -6.62437 & 4.627042 & 0.798223 \\ -9.68989 & 5.499808 & -0.49785 \\ -5.48543 & 5.103742 & 1.457551 \\ -10.0262 & 6.206262 & 0.663212 \\ -7.08061 & 3.331800 & 0.967942 \\ -8.44241 & 4.962350 & -0.72177 \\ -4.19742 & 6.796978 & 1.803308 \\ -11.5679 & 7.249062 & 1.747576 \\ -5.52366 & 8.331362 & 0.336581 \\ -13.3083 & 6.867680 & -0.01583 \\ -7.55952 & 7.464959 & -0.83586 \\ -12.7085 & 5.594786 & -2.08021 \\ -4.92303 & 4.423742 & 2.098670 \\ -9.26279 & 6.360781 & 1.426439 \\ -6.80858 & 12.86786 & -0.22620 \\ -6.19246 & 1.992006 & 0.662521 \\ -3.86631 & 1.920459 & 0.147225 \\ -1.55061 & 1.82810 & -0.34515\end{array}$




$\begin{array}{llll}\mathrm{O} & -4.75726 & 1.853092 & 1.562655 \\ \mathrm{O} & -2.65278 & 3.062350 & -0.00228 \\ \mathrm{O} & -5.26778 & 2.046873 & -0.75597 \\ \mathrm{O} & -2.78634 & 0.686154 & -0.19312 \\ \mathrm{O} & -0.39861 & 1.530373 & 0.783755 \\ \mathrm{H} & 0.401761 & 2.077850 & 0.750081 \\ \mathrm{O} & -7.25640 & 0.770717 & 0.824931 \\ \mathrm{H} & -6.89573 & -0.12707 & 0.752283 \\ \mathrm{O} & -0.92733 & 2.058559 & -1.84346 \\ \mathrm{H} & -0.23530 & 1.443647 & -2.13371 \\ \mathrm{Cl} & -5.74570 & 12.62116 & -0.91322 \\ \mathrm{Cl} & -10.2706 & 4.396204 & -2.92497\end{array}$

$\begin{array}{llll}\text { M7 } & & & \\ \mathrm{C} & -5.50159 & 6.619321 & -0.14527 \\ \mathrm{C} & -5.86483 & 7.200709 & 1.074135 \\ \mathrm{C} & -6.77293 & 6.552624 & 1.916936 \\ \mathrm{C} & -7.31129 & 5.319938 & 1.541509 \\ \mathrm{C} & -6.96002 & 4.739925 & 0.311382 \\ \mathrm{C} & -6.05386 & 5.394879 & -0.52954 \\ \mathrm{O} & -7.53708 & 3.538488 & -0.06415 \\ \mathrm{H} & -4.78532 & 7.115738 & -0.80168 \\ \mathrm{H} & -5.44199 & 8.159196 & 1.379534 \\ \mathrm{H} & -7.06657 & 6.988238 & 2.871998 \\ \mathrm{H} & -5.7873 & 4.922954 & -1.47662 \\ \mathrm{Si} & -6.6526 & 2.156967 & -0.11477 \\ \mathrm{Si} & -4.28095 & 2.031386 & 0.079156 \\ \mathrm{Si} & -1.92293 & 1.900124 & 0.267766 \\ \mathrm{O} & -5.55456 & 1.993002 & 1.163148 \\ \mathrm{O} & -3.05352 & 3.154861 & 0.259471 \\ \mathrm{O} & -5.34983 & 2.174211 & -1.20318 \\ \mathrm{O} & -3.17289 & 0.77739 & 0.077268 \\ \mathrm{O} & -1.14386 & 1.604324 & 1.679316 \\ \mathrm{H} & -0.37109 & 2.15542 & 1.880644 \\ \mathrm{O} & -7.7579 & 0.984881 & -0.33722 \\ \mathrm{H} & -7.41499 & 0.095899 & -0.52077 \\ \mathrm{O} & -0.89552 & 2.092276 & -0.99588 \\ \mathrm{H} & -0.15155 & 1.472059 & -1.05557 \\ \mathrm{Cl} & -8.40015 & 4.482812 & 2.611871 \\ \mathrm{C} & -14.7338 & 5.363564 & 0.553307 \\ \mathrm{C} & -14.3469 & 6.462146 & -0.22439 \\ \mathrm{C} & -12.9902 & 6.68782 & -0.47194 \\ \mathrm{C} & -12.0357 & 5.817327 & 0.054844 \\ \mathrm{C} & -12.4104 & 4.710081 & 0.840949 \\ \mathrm{C} & -13.7772 & 4.495626 & 1.078515\end{array}$




$\begin{array}{llll}\mathrm{O} & -11.5213 & 3.837358 & 1.381655 \\ \mathrm{H} & -15.7908 & 5.17852 & 0.751749 \\ \mathrm{H} & -15.0921 & 7.141778 & -0.63968 \\ \mathrm{H} & -12.6614 & 7.533521 & -1.07714 \\ \mathrm{H} & -14.0608 & 3.635170 & 1.686865 \\ \mathrm{H} & -10.6100 & 4.136276 & 1.179317 \\ \mathrm{Cl} & -10.3293 & 6.091669 & -0.23906\end{array}$

TS4

$\begin{array}{llll}\mathrm{C} & -5.48144 & 6.584055 & 0.718317 \\ \mathrm{C} & -6.56115 & 7.453074 & 0.883634 \\ \mathrm{C} & -7.86147 & 6.948823 & 0.854824 \\ \mathrm{C} & -8.13825 & 5.600653 & 0.584724 \\ \mathrm{C} & -7.02662 & 4.720030 & 0.576206 \\ \mathrm{C} & -5.71490 & 5.203151 & 0.651009 \\ \mathrm{O} & -7.26399 & 3.353740 & 0.500025 \\ \mathrm{H} & -4.47426 & 6.998758 & 0.617440 \\ \mathrm{H} & -6.38404 & 8.525845 & 1.001798 \\ \mathrm{H} & -8.70836 & 7.606202 & 1.065505 \\ \mathrm{H} & -4.87971 & 4.508208 & 0.638495 \\ \mathrm{Si} & -6.34528 & 1.990264 & 0.415361 \\ \mathrm{Si} & -3.98336 & 1.656278 & 0.105626 \\ \mathrm{Si} & -1.65914 & 1.374129 & -0.27793 \\ \mathrm{O} & -4.98086 & 1.825782 & 1.438347 \\ \mathrm{O} & -2.68392 & 2.697387 & -0.04113 \\ \mathrm{O} & -5.29818 & 1.825519 & -0.91546 \\ \mathrm{O} & -2.97833 & 0.328091 & -0.07881 \\ \mathrm{O} & -0.51195 & 1.066096 & 0.853744 \\ \mathrm{H} & 0.330073 & 1.564144 & 0.779773 \\ \mathrm{O} & -7.49672 & 0.840689 & 0.607398 \\ \mathrm{H} & -7.16728 & -0.05421 & 0.831480 \\ \mathrm{O} & -1.00782 & 1.489676 & -1.77634 \\ \mathrm{H} & -0.21342 & 0.945829 & -1.96330 \\ \mathrm{Cl} & -7.11116 & 4.592813 & 4.306052 \\ \mathrm{C} & -12.2259 & 4.794622 & -0.61635 \\ \mathrm{C} & -11.4497 & 5.754943 & -1.27627 \\ \mathrm{C} & -10.135 & 5.987352 & -0.86850 \\ \mathrm{C} & -9.53928 & 5.251754 & 0.178118 \\ \mathrm{C} & -10.3456 & 4.286234 & 0.825771 \\ \mathrm{C} & -11.6836 & 4.062173 & 0.429688 \\ \mathrm{O} & -9.90379 & 3.528280 & 1.857785 \\ \mathrm{H} & -13.2695 & 4.613480 & -0.90261 \\ \mathrm{H} & -11.8887 & 6.320313 & -2.11217 \\ \mathrm{H} & -9.52775 & 6.735100 & -1.38842 \\ \mathrm{H} & -12.2666 & 3.318750 & 0.982634\end{array}$




$\begin{array}{llll}\mathrm{H} & -8.93735 & 3.687700 & 1.951298 \\ \mathrm{Cl} & -8.75155 & 5.882132 & 4.507390\end{array}$

M8

C

C

C

C

C

C

$\mathrm{O}$

$\mathrm{H}$

$\mathrm{H}$

$\mathrm{H}$

$\mathrm{H}$

$\mathrm{Si}$

$\mathrm{Si}$

$\mathrm{Si}$

O

$\mathrm{O}$

$\mathrm{O}$

$\mathrm{O}$

$\mathrm{O}$

$\mathrm{H}$

$\mathrm{O}$

$\mathrm{H}$

$\mathrm{O}$

$\mathrm{H}$

$\mathrm{Cl}$

C

C

C

C

C

C

$\mathrm{O}$

$\mathrm{H}$

$\mathrm{H}$

$\mathrm{H}$

$\mathrm{H}$

$\mathrm{H}$

$\mathrm{Cl}$

$\begin{array}{lll}-4.72866 & 6.241034 & 1.563612 \\ -5.51798 & 7.063422 & 0.752535 \\ -6.73781 & 6.595804 & 0.265415 \\ -7.2085 & 5.298305 & 0.555289 \\ -6.38743 & 4.504021 & 1.376294 \\ -5.17006 & 4.954893 & 1.881770 \\ -6.83686 & 3.22098 & 1.710027 \\ -3.77674 & 6.595419 & 1.960334 \\ -5.19265 & 8.076956 & 0.515092 \\ -7.36381 & 7.245108 & -0.34794 \\ -4.5905 & 4.296142 & 2.528088 \\ -6.06141 & 1.848312 & 1.242373 \\ -3.8894 & 1.761502 & 0.273183 \\ -1.71399 & 1.664855 & -0.65785 \\ -4.48588 & 1.670886 & 1.836516 \\ -2.72101 & 2.900077 & -0.09391 \\ -5.4441 & 1.913767 & -0.33009 \\ -2.91152 & 0.528184 & -0.29330 \\ -0.37425 & 1.336084 & 0.227534 \\ 0.398487 & 1.906804 & 0.091139 \\ -7.12358 & 0.674713 & 1.613044 \\ -6.81061 & -0.24092 & 1.540761 \\ -1.38782 & 1.916207 & -2.24386 \\ -0.73616 & 1.329025 & -2.65865 \\ -5.85818 & 4.380002 & 5.392317 \\ -10.9247 & 4.207556 & -1.34444 \\ -9.89911 & 4.84182 & -2.06043 \\ -8.70133 & 5.146936 & -1.41433 \\ -8.47337 & 4.833615 & -0.05849 \\ -9.51726 & 4.164787 & 0.642516 \\ -10.7322 & 3.874538 & -0.00872 \\ -9.46053 & 3.821927 & 1.955634 \\ -11.87 & 3.964843 & -1.83341 \\ -10.0286 & 5.089595 & -3.11481 \\ -7.89911 & 5.635848 & -1.96947 \\ -11.509 & 3.375751 & 0.572222 \\ -8.52855 & 3.651227 & 2.214653 \\ -7.74288 & 4.905145 & 4.624185\end{array}$


1. Nganai, S.; Lomnicki, S.; Dellinger, B., Formation of PCDD/Fs from oxidation of 2monochlorophenol over an $\mathrm{Fe}_{2} \mathrm{O}_{3}$ /silica surface Chemosphere 2012, 88, 371-376.

2. Lomnicki, S.; Dellinger, B., A detailed mechanism of the surface-mediated formation of PCDD/F from the oxidation of 2-chlorophenol on a CuO/silica surface J. Phys. Chem. A. 2003, 107, 4387-4395. 